BNL-113560-2017-JA

\title{
Modified Inverse Micelle Synthesis for Mesoporous Alumina with a High D4 Siloxane Adsorption Capacity
}

\author{
Wei Zhong, Ting Jiang, Tahereh Jafari, Altug S. Poyraz, \\ Wei Wu, David A. Kriz, Shoucheng Du, Sourav Biswas, \\ Michael Thompson Pettes, and Steven L. Suib
}

Submitted to Microporous and Mesoporous Materials

February 2017

\author{
Energy and Photon Sciences Directorate \\ Brookhaven National Laboratory
}

\author{
U.S. Department of Energy \\ USDOE Office of Science (SC), \\ Basic Energy Sciences (BES) (SC-22)
}

\footnotetext{
Notice: This manuscript has been co-authored by employees of Brookhaven Science Associates, LLC under Contract No. DE-SC0012704 with the U.S. Department of Energy. The publisher by accepting the manuscript for publication acknowledges that the United States Government retains a non-exclusive, paid-up, irrevocable, world-wide license to publish or reproduce the published form of this manuscript, or allow others to do so, for United States Government purposes.
} 


\section{DISCLAIMER}

This report was prepared as an account of work sponsored by an agency of the United States Government. Neither the United States Government nor any agency thereof, nor any of their employees, nor any of their contractors, subcontractors, or their employees, makes any warranty, express or implied, or assumes any legal liability or responsibility for the accuracy, completeness, or any third party's use or the results of such use of any information, apparatus, product, or process disclosed, or represents that its use would not infringe privately owned rights. Reference herein to any specific commercial product, process, or service by trade name, trademark, manufacturer, or otherwise, does not necessarily constitute or imply its endorsement, recommendation, or favoring by the United States Government or any agency thereof or its contractors or subcontractors. The views and opinions of authors expressed herein do not necessarily state or reflect those of the United States Government or any agency thereof. 


\section{Modified inverse micelle synthesis for mesoporous alumina with a high D4 siloxane adsorption capacity}

Wei Zhong, ${ }^{\text {a }}$ Ting Jiang, ${ }^{\mathrm{b}}$ Tahereh Jafari, ${ }^{\mathrm{a}}$ Altug S. Poyraz, ${ }^{\mathrm{c}}$ Wei Wu, ${ }^{\mathrm{a}, \mathrm{d}}$ David A. Kriz, ${ }^{\mathrm{e}}$ Shoucheng Du, Sourav Biswas, ${ }^{\mathrm{e}}$ Michael Thompson Pettes ${ }^{\mathrm{a}, \mathrm{d}}$ and Steven L. Suib ${ }^{\text {*a,b,e }}$

a. Institute of Materials Science, University of Connecticut, U-3136, 97 North Eagleville Rd., Storrs, Connecticut 06269 (USA).

b. Department of Chemical \& Biomolecular Engineering, University of Connecticut, U-3222, 191 Auditorium Rd., Storrs, Connecticut 06269 (USA).

c. Brookhaven National Laboratory, Upton, New York 11973(USA).

d. Department of Mechanical Engineering, University of Connecticut, U- 3139, 191 Auditorium Rd., Storrs, Connecticut 06269(USA).

e.Department of Chemistry, University of Connecticut, U-3060, 55 North Eagleville Rd., Storrs, Connecticut 06269 (USA). 
Abstract: In this work, mesoporous aluminas (MAs) with uniform and monomodal pores were fabricated via a modified inverse micelle synthesis method, using a non-polar solvent (to minimize the effect of water content) and short reaction time (for a fast evaporation process). The effects of reaction times (4-8 h), surfactant chain lengths (non-ionic surfactants), and calcination temperatures and hold times (450-600 ${ }^{\circ} \mathrm{C} ; 1-4 \mathrm{~h}$ ) on the textural properties of MA were studied. The targeted pore sizes of MA were obtained in the range of 3.1-5.4 nm by adjusting the surfactant and reaction time. The surface area and pore volume were controlled by the calcination temperature and hold time while maintaining the thermal stability of the materials. The tuned MA of the large mesopore volume achieved $168 \mathrm{mg} / \mathrm{g}$ octamethylcyclotetrasiloxane (D4 siloxane) adsorption capacity, a 32\% improvement compared to commercially activated alumina. After three adsorption recycles, the synthesized MA still maintained approximate $85 \%$ of its original adsorption capacity, demonstrating a sustainable adsorption performance and high potential for related industrial applications.

Keywords: Mesoporous alumina (MA); reaction times; surfactant chain lengths; calcination temperatures and hold times; textural properties; D4 siloxane; adsorption. 


\section{Introduction}

Biogas (primarily methane and carbon dioxide) produced by anaerobic digestion of waste materials possesses considerable energy value. Traces of impurities in the biogas (e.g., sulfides, halides, and siloxane compounds) need to be removed before use.[1-7] Siloxane, one of the impurities, refers to a subgroup of silicones containing $\mathrm{Si}-\mathrm{O}$ bonds with organic chains attached to the silicon atom.[1] During biogas combustion, siloxane, if not removed beforehand, can form abrasive microcrystalline silica particles which inhibit heat conduction and lubrication of the engine.[2,8-10]Current technologies for siloxane removal from biogas are mainly based on adsorption. Adsorbents cover a variety of materials such as activated carbons (ACs), zeolites, molecular sieves, or silica gels.[1,2,11] The most widely used adsorbents are ACs,[12] but regeneration of ACs is difficult due to the polymerization of adsorbed siloxanes on the AC surface. $[1,5,13]$

Mesoporous materials, especially MAs, [14-18] are considered promising for siloxane adsorption because of their proper pore diameters, $[1,15]$ and high surface areas. In our previous work, mesoporous aluminosilicates prepared by the same synthetic strategy showed improved adsorption performance with higher aluminum concentrations. As for mesoporous aluminosilicates, surface area and pore volume are two key factors that strongly influence the adsorption capacity of D4 siloxane.[19] Apart from the goal of high pore volumes, pore size is another particularly significant textural property to consider. Porous materials with continuously adjustable pore sizes have attracted great interest in a variety of applications such as molecular sieves, selective adsorption, and sizeselective catalysis.[20,21] All of these have led us to develop MA materials with adjustable pore sizes and high pore volumes. 
Different synthetic methods have been employed for preparing MA, such as an aerosol generation method using block copolymers, a modified sol-gel method with organic templates, and an evaporation-induced self-assembly (EISA).[22,23] The EISA strategy is a significant achievement for synthesizing ordered MA (Table S1). However, there is a continual dispute on the importance of ordered versus wormhole-like pore structures. It is desired to develop both pore structure MA to meet the requirements of various applications.[24,25]As for wormhole-like pore structure MA, the common synthetic pathways deduced from the synthesis methods of mesoporous silica yield unsatisfactory alumina materials.[20] Examples of the unadjustable pore size for wormhole-like MA synthesized by the currently available methods can be found from Vaudry et al., who reported MA materials with a narrow pore size distribution prepared by anionic surfactants (carboxylic acids). [26] And Kim et al. synthesized MA of identical pore size $(4 \mathrm{~nm})$ irrespective of the variation of surfactant/aluminum precursor ratio.[27]

The main contribution of this research is to explore controllable wormhole-like MA synthesis. As shown in Table S2, the water content is critical during the synthesis. Therefore the reagent to water ratio has to be strictly controlled.[28] Our synthetic strategy includes a non-polar solvent to minimize the effects of water and create a fast evaporation process. This strategy has been successfully used for preparing different mesoporous transition metal oxides, such as manganese oxide,[29,30] cobalt oxide,[31] titanium oxide,[32,33] and iron oxide[34,35], which displayed superior performances in different applications. However, this is the first time this synthetic strategy has been used for the preparation of wormhole-like MA (Table S2). The synthesis parameters have been modified to develop this synthetic strategy further. It is important and significant to learn more about this synthetic strategy through this study, which may be applied to other mesoporous systems. Reaction times, surfactant chain lengths and calcination temperatures, which are commonly used for adjusting 
pore sizes[20,36-38], were chosen as parameters for adjusting the textural properties (pore sizes, surface areas, and pore volumes) of alumina. The synthesized MAs have been demonstrated to have an excellent D4 siloxane adsorption capacity, compared with commercially activated alumina. Some insights to correlate synthesis parameters with the material textural properties and adsorption performance are also involved in this study.

\section{Experimental}

\subsection{Synthesis of MA.}

All chemicals were used as received without further purification. During the synthesis, $5 \mathrm{~g}$ aluminum sec-butoxide (Alfa-Aesar) was diluted in $10 \mathrm{~g}$ 1-butanol (Sigma-Aldrich) with stirring at ambient temperature. Then 2 g Pluronic P123 (Poly (ethylene glycol)-block-Poly(propylene glycol)block-Poly(ethylene glycol); $\mathrm{PEO}_{20}-\mathrm{PPO}_{70}-\mathrm{PEO}_{20}$; Sigma-Aldrich) was added. At the same time, $2 \mathrm{~g}$ nitric acid (J.T.Baker) was dissolved in $10 \mathrm{~g}$ 1-butanol. The two solutions reacted at $120{ }^{\circ} \mathrm{C}$ for $6 \mathrm{~h}$, yielding a transparent gel. The gel was washed several times with ethanol to remove surfactants and dried in a vacuum oven at room temperature overnight. Different reaction times $(4 \mathrm{~h}, 6 \mathrm{~h}, 8 \mathrm{~h})$ were studied at $120{ }^{\circ} \mathrm{C}$ with P123 as the surfactant. After being ground, the obtained powders were placed in a cuvette and calcined in air at $450-600{ }^{\circ} \mathrm{C}$ for $1-4 \mathrm{~h}$, with a $2{ }^{\circ} \mathrm{C} / \mathrm{min}$ heating rate. $0.2 \mathrm{~g}$ Brij56 $\left(\mathrm{C}_{16} \mathrm{H}_{33}(\mathrm{PEO})_{10} \mathrm{OH}\right.$; Sigma-Aldrich), and 4.3 g F127 ( $\mathrm{PEO}_{106} \mathrm{PPO}_{70} \mathrm{PEO}_{106}$; Sigma-Aldrich) were also investigated to show the effect of surfactant chain lengths. Surfactant F127 has a longer chain length of PEO block than that of P123. Compared to the Pluronic triblock copolymers, Brij56 surfactant is a much shorter diblock polymer composed of a hydrophilic PEO block and $\mathrm{C}_{16} \mathrm{H}_{33}$ alkyl chains. The obtained powders were calcined in air at $500{ }^{\circ} \mathrm{C}$ for $1 \mathrm{~h}$, with a $2{ }^{\circ} \mathrm{C} / \mathrm{min}$ heating rate. The synthesis parameters for different samples are recorded in Table S4. After calcination, the 
samples were stored in screw-cap vials and put in a vacuum oven before usage to desorb water and weakly held contaminants. [17] The samples were packed in the reactor for D4 siloxane adsorption immediately after being removed from the vacuum oven.

\subsection{Characterization.}

X-ray diffraction (XRD) analyses were performed in a Rigaku Ultima IV diffractometer $(\mathrm{Cu} \mathrm{K} \alpha$ radiation, $\lambda=1.5406 \AA$ ) with an operating voltage of $40 \mathrm{kV}$ and a current of $44 \mathrm{~mA}$. The low angle PXRD patterns were collected over a 2 -theta range of $0.5-10^{\circ}$ with a continuous scan rate of $0.5^{\circ}$ min. The wide-angle PXRD patterns were collected over a 2-theta range of $10-80^{\circ}$ with a continuous scan rate of $2.0^{\circ} / \mathrm{min}$. Nitrogen sorption experiments were conducted in a Quantachrome Autosorb-1$1 \mathrm{C}$ automated adsorption system. The powders were degassed for $6 \mathrm{~h}$ at $150{ }^{\circ} \mathrm{C}$. Surface areas were calculated using the Brunauer-Emmett-Teller (BET) method. Pore size distributions and pore volumes were calculated using the Barrett-Joyner-Halenda (BJH) method from the desorption isotherm. Morphological characterization was done using an FEI Teneo LoVac FEG-SEM. Transmission electron microscopy (TEM) images of synthesized mesoporous materials were recorded on a JEOL 2010 FasTEM microscope operated at $200 \mathrm{kV} . \mathrm{CO}_{2}$ temperature-programmed desorption (TPD) was conducted using a Netzsch TG 209 F1 Libra thermogravimetric analyzer coupled to a QMS 403C quadrupole mass spectrometer. The samples were pretreated at $200{ }^{\circ} \mathrm{C}$ for 1 h to clean the sample surface before each test. $\mathrm{CO}_{2}$ adsorption was performed at $27{ }^{\circ} \mathrm{C}$ with 50 $\mathrm{cm}^{3} / \min 5 \% \mathrm{CO}_{2}$ balanced in argon for $1 \mathrm{~h}$. Physisorbed $\mathrm{CO}_{2}$ was removed by flowing argon for 30 min. After that, the samples were heated from $27{ }^{\circ} \mathrm{C}$ to $900{ }^{\circ} \mathrm{C}$ under $15{ }^{\circ} \mathrm{C} / \mathrm{min}$ in argon. Pyridine adsorption studies were performed with $13 \mathrm{~mm}$ diameter self-supporting pellets using a Nicolet Magna 560 FTIR. The pellets were cleaned at $250{ }^{\circ} \mathrm{C}$ overnight. A $1 \mathrm{M}$ pyridine solution in methanol 
was dropped on the pellets, and physisorbed pyridine was removed at $200{ }^{\circ} \mathrm{C}$ for $90 \mathrm{~min}$. The spent adsorbent A1120-8h after the first run was analyzed using a Bruker Avance III 400 MHz solid-state magic-angle spinning nuclear magnetic resonance (MAS NMR) spectroscopy, and a PHI Model 590 X-ray photoelectron spectroscopy (XPS).

\subsection{D4 siloxane adsorption.}

Each D4 siloxane (Sigma-Aldrich) adsorption test was performed over $100 \mathrm{mg}$ of adsorbent material at room temperature and atmospheric pressure in a tubular quartz reactor $(15 \mathrm{~cm}$ long, 0.6 $\mathrm{cm}$ internal diameter). D4 siloxane was introduced with a nitrogen carrier gas flow (15 $\mathrm{mL} / \mathrm{min})$. The D4 siloxane amount in the carrier gas was approximately $6.4 \mathrm{mg} / 60 \mathrm{~min}$. After passing through the adsorbents, a gas wash bottle filled with $200 \mathrm{~mL}$ hexane (Sigma-Aldrich) was used to trap the residual siloxane in the carrier gas. The D4 siloxane concentration in the solvent trap was sampled every 20 min and measured in an Agilent gas chromatography/ mass selective detector (GC/MSD) system (GC 7820A and MSD 5975) equipped with an HP-1 capillary column $(12 \mathrm{~m} \times 200 \mu \mathrm{m} \times 0.33$ $\mu \mathrm{m})$. Calibration was carried out using D4 siloxane standards.

Breakthrough time was identified from breakthrough curves, and then breakthrough capacity was obtained from the corresponding saturation curve. The saturation curve was obtained by subtraction of the breakthrough curve from the blank breakthrough curve (tested with no adsorbents), which presented the D4 amount adsorbed. Saturation capacity is the maximum adsorption capacity. The spent adsorbent was recovered after the adsorption tests, mixed with $50 \mathrm{~mL}$ of hexane, and centrifuged after sonication to extract the adsorbed siloxane from the adsorbent. The extract was then analyzed in the GC/MSD system to obtain the wash capacity of the adsorbents, which was identical 
to its saturation capacity. The adsorbent after extraction was dried at $100{ }^{\circ} \mathrm{C}$ overnight and tested again for evaluation of its stability.

\section{Results}

\subsection{Modification of MA pore size by adjusting the reaction time and surfactant.}

All the MA materials in Figure 1a show one broad XRD line in the low angle region, and the wall thicknesses are calculated accordingly (Table 1). There is only one peak in the low angle XRD range, indicating a nano-sized periodicity and uniform mesoporosity.[24,39] The peak positions are $9.3 \mathrm{~nm}$, $11.8 \mathrm{~nm}$, and $14.7 \mathrm{~nm}$ with the reaction times of $4 \mathrm{~h}, 6 \mathrm{~h}$, and $8 \mathrm{~h}$, respectively, which indicates the pore size increases with the reaction time. The peak positions are $9.8 \mathrm{~nm}, 11.8 \mathrm{~nm}$, and $14.7 \mathrm{~nm}$ with surfactants of Brij56, P123, and F127, respectively, which indicates that the pore size increases with the chain lengths of the surfactants. The wide-angle XRD patterns of these samples (Figure S3) reflect their low crystallinity. The sample Al120-8h is identified as $\gamma$-alumina (JCPDS\#:10-0425) after subsequent thermal treatment up to $900{ }^{\circ} \mathrm{C}$ for $1 \mathrm{~h}$ (Figure $\mathrm{S} 4$ ).

Mesoporosity of the alumina is further illustrated by the $\mathrm{N}_{2}$ adsorption/desorption isotherms and BJH desorption pore size distributions (Figure 1b, c). All alumina samples show a similar welldefined step in the adsorption isotherm (Type IV isotherm), indicating a normal mesoporous structure,[36] and a hysteresis in the desorption isotherm over the same relative pressure range (H1 hysteresis loop), suggesting the formation of cylindrical pores.[24] Figure $1 \mathrm{~b}$ shows that the capillary condensation steps for samples Al120-4h, Al120-6h, and Al120-8h shift slightly to the greater relative pressures, indicating an increase in the size of mesopores with the increasing reaction time. A similar trend is also observed for samples Al-Brij56, Al-P123 (A1120-6h), and Al-F127, indicating an increase in the mesopore size with the chain lengths of the surfactants increasing. In Figure 1c, the pore diameters of the mesoporous Al120-4h, Al120-6h, and Al120-8h are observed to increase with 
the reaction time increasing. The pore diameter of mesoporous Al-F127 is larger than that of AlP123, while the pore size of Al-Brij 56 is the smallest among the three samples with different surfactants as the variable parameter (Figure 1c).

The TEM images of the representative samples Al120-6h and Al120-8h (Figure 1d) show that no apparent order in the pore arrangement exists, which is in good agreement with the low angle XRD patterns. The pore packing motif can be described as wormhole-like.[20,40] Though regular in average diameter, the wormhole-like channels have no long-range order. That is, the channels appear to be packed randomly. The low angle XRD line may result from the regular separation between single channel walls.[40] The pore sizes are shown to be improved with the reaction time increasing from $6 \mathrm{~h}$ to $8 \mathrm{~h}$.

\subsection{Modification of MA surface area and pore volume by adjusting the calcination} temperature and hold time.

Mesoporosity is maintained at different calcination temperatures and hold times as shown in the low angle XRD patterns (Figure 2a). All of the resulting MA materials have low crystallinity (Figure S3), and All of the as prepared samples-show mesoporous characteristic Type IV isotherms and monomodal pore size distributions regardless of the calcination temperature or hold time (Figure $2 b, c)$. As shown in Table 1 , the surface area and pore volume increase when the calcination temperature is increased from $450{ }^{\circ} \mathrm{C}$ to $600{ }^{\circ} \mathrm{C}$, whereas the pore size does not have an obvious change with an increasing calcination temperature. (The evidence of organic template removal is provided in Figures S5 and S6.) The surface area and pore volume also increase with an increase of the calcination hold time, though the hold time has no obvious effect on the pore size of MA. All of these results signify that a good control of the surface area and pore volume of MA is achieved by 
adjusting the heat treatment parameters. The high-resolution TEM image (Figure 2d) of Al500-1h (A1120-8h) shows a highly interconnected pore system.

\subsection{D4 siloxane adsorption.}

The reaction time is a major factor in the synthesis method to adjust the pore volumes according to the summary in Table1, so Al120-6h and Al120-8h are chosen for D4 siloxane adsorption tests. Commercially activated alumina is the most well-known alumina sample for adsorption or catalysis. Figure 3 shows a comparison of D4 siloxane adsorption tests of the as-synthesized samples (A11206h, Al120-8h) and commercially activated alumina. As revealed from the D4 breakthrough curve in Figure 3a, D4 does not break through Al120-8h until 100 min (breakthrough capacity: 45 $\mathrm{mg} / \mathrm{g}$ ). However, commercially activated alumina and Al120-6h have breakthroughs at approximately $60 \mathrm{~min}$ (breakthrough capacity: $\sim 8 \mathrm{mg} / \mathrm{g}$ ). That means the breakthrough capacity of Al120-8h is approximate 5.6 times greater than that of commercially activated alumina. According to the D4 saturation curve (Figure 3b), the saturation capacity of commercially activated alumina is 127 $\mathrm{mg} / \mathrm{g}$. For Al120-8h, the saturation capacity is $168 \mathrm{mg} / \mathrm{g}$, indicating an improvement of $32 \%$ compared to that of commercially activated alumina. The surface area and pore volume of commercially activated alumina are recorded in Table $1\left(\mathrm{~N}_{2}\right.$ sorption related measurements are shown in Figure S8).

\subsection{Adsorbent regeneration.}

MA Al120-8h can be easily regenerated. The wash capacities of the adsorbents are 162, 148, 143 $\mathrm{mg} / \mathrm{g}$ from the first (1st) to third (3rd) recycle, respectively, which maintains $96 \%$, $88 \%$, and $85 \%$ capacity of the fresh adsorbent (Figure 4a). Wide scan XPS data (Figure 4b) reveal an atomic ratio of 
$\mathrm{Si} / \mathrm{Al} \sim 0.4$ on the surface of the spent Al120-8h after one run. In contrast, the most widely used adsorbent ACs are not easily recovered even at high temperatures (250-300 ${ }^{\circ} \mathrm{C}$ ) for an extended period.[41] The capacity loss is an effect related to the polymerization of D4 on the surface of ACs due to the phenolic and carboxylic groups on their surfaces. A 96\% capacity is maintained for the first recycle of Al120-8h, indicating that a polymerization phenomenon is not evident after one D4 siloxane adsorption run, which is further verified by ${ }^{29} \mathrm{Si}$ MAS NMR (Figure 4c) and ATR (Figure 4d) measurements on spent Al120-8h after one run.

Since only one peak at $\delta=-19$ is observed in the ${ }^{29} \mathrm{Si}$ MAS NMR spectrum for spent Al120-8h after one run (Figure 4c), and NMR peaks for other siloxanes are not observed, which indicates that there is no obvious polymerization on the Al120-8h adsorbent.[42] The ATR spectrum of spent Al120-8h recorded after one D4 siloxane adsorption run is compared with the spectra of the fresh adsorbent and D4 (Figure 4d). Bands at wavenumbers of 1255, 1062, and $804 \mathrm{~cm}^{-1}$ are detected for spent Al120-8h, which are ascribed to the $\mathrm{Si}-\mathrm{CH}_{3}$ bond, the asymmetric, and the symmetric stretches of the $\mathrm{Si}-\mathrm{O}-\mathrm{Si}$ bridge bonds of D4 siloxane, respectively.[41] There are no other bands, and thus no polymerization for A1120-8h after one run is observed.[43] After recycling for three times, the adsorbent shows excellent stability as compared to the significant loss of capacity for ACs and silica gel. $[1,43,44]$

\section{Discussion}

\subsection{Formation of MA.}

Inverse micelles are formed as nanoreactors by surfactants as shown in Scheme 1. Aluminum oxo-clusters are stabilized in hydrated inverse micelles at a low $\mathrm{pH}$ and interact with surfactant via a charge transfer interaction or hydrogen bonding. The aluminum precursor loaded inverse micelles are 
packed randomly during the reaction at $120{ }^{\circ} \mathrm{C}$. A concomitant color change to yellow after reaction for approximately $20 \mathrm{~min}$ indicates the formation of $\mathrm{NO}_{x}$ (a wide range of nitric oxides), which is proved during the synthesis of other mesoporous oxides prepared based on the same synthetic strategy (e.g., silica, titania, manganese oxides).[36] Packing is followed by oxidation and condensation of the aluminum precursors in the micelles. Surfactant species are oxidized to form carboxyl groups coordinated to aluminum oxo-clusters, which is observed for transition metal systems.[36] $\mathrm{MA}$ is formed as $\mathrm{pH}$ increases due to $\mathrm{NO}_{x}$ formation from thermal decomposition of nitrate ions during the reaction. Finally, the residual surfactants are washed off, and surface $\mathrm{NO}_{x}$ and carboxyl species are removed after calcination. The crystalline walls of as-synthesized mesoporous materials are made up of nanoparticles of alumina, and the mesopores are the intra-connecting voids formed between randomly packed nanocrystals.[36] The hydrolysis and condensation rates of aluminum alkoxides are generally faster than that of silicon alkoxides,[21] so synthesis methods used for mesoporous silica have not always yielded satisfactory results for MA. As for our synthesis strategy, the rates of hydrolysis and condensation of aluminum precursors are controlled by in situ formation of $\mathrm{NO}_{x}$ from nitrate ions.

\subsection{Effect of synthesis parameters on textural properties of MA.}

The fact that a longer reaction time results in a larger pore size is attributed to the longer condensation time for the precursors. The more precursors condense during a longer reaction time, and the more inverse micelles are packed together to form a bigger mesopore and a larger pore volume, which is confirmed by the change of the gel color and the wall thickness. The as-formed gel is white after $4 \mathrm{~h}$, which turns yellow after $6 \mathrm{~h}$ and golden yellow after $8 \mathrm{~h}$ (Figure S2). The gel adsorbs $\mathrm{NO}_{x}$ during the reaction, and the color darkens because more $\mathrm{NO}_{x}$ is formed, which indicates 
more precursors participate in the reaction. The wall thickness increases by approximately $68 \%$ from the reaction time of $4 \mathrm{~h}$ to $8 \mathrm{~h}$. At the same time, the pore size increases by approximately $39 \%$.

There is no need for a stringent selection of surfactants to prepare MA with this facile method. MA with a high surface area and narrow pore size distribution are obtained with non-ionic surfactants like Brij56, P123, and F127. Furthermore, pore sizes of MA are continuously adjusted by choosing surfactants with different chain lengths of the PEO block. The chain lengths of the PEO blocks of F127 and P123 surfactants are essential in determining the pore size and pore volume of MA,[45] with the central PPO blocks having the same length. The PEO block chain length of F127 is much longer than that of P123, and the pore size and pore volume of Al-F127 are larger than that of Al-P123. The unit number of PEO block of Brij56 is considerably smaller than that of P123 or F127, which generates Al-Brij56 with a smaller pore size and pore volume. The sizes of inverse surfactant micelle nanoreactors get bigger with longer surfactants, more inverse micelles pack together, and therefore interstitial voids (pores) formed in between the alumina nanoparticles get larger. PEO block is expected to be more closely associated with the inorganic alumina wall than the more hydrophobic PPO block because the PEO block interacts with the alumina species more strongly through a charge transfer interaction or hydrogen bonding.[37] Therefore, the wall thickness increases as expected by approximately $41 \%$ from surfactant Brij56 to F127 with the increasing length of the PEO block. The pore size increases by approximately 69\%. The surfactant chain length has a more potent effect on increasing the pore size than the reaction time does. Calcination temperature and hold time have no obvious effect on the pore sizes of as-synthesized MA, which shows the pore structures have good thermal stability under these heat treatment conditions.

\subsection{Effect of textural properties on D4 siloxane adsorption and adsorbent regeneration.}


Since the pore sizes of all the as-synthesized MA samples are larger than the molecular size of D4, pore size is not a limiting parameter for the MA adsorbents. Cabrera-Codony et al. reported that adsorption is strongly related to the textural properties of ACs, and the pore volume plays a key role in D4 siloxane adsorption,[1] which is in agreement with our previous work on mesoporous aluminosilicates.[19] Pore volume is also a key factor in our system. As presented in Table 1, although the pore size and surface area of Al120-6h are larger than that of commercially activated alumina, the total pore volume of Al120-6h $\left(0.20 \mathrm{cc} \mathrm{g}^{-1}\right)$ is less than that of commercially activated alumina $\left(0.26 \mathrm{cc} \mathrm{g}^{-1}\right)$. The saturation capacity of Al120-6h $(92 \mathrm{mg} / \mathrm{g})$ is $28 \%$ less than that of commercially activated alumina. All textural parameters of Al120-8h are larger than that of commercially activated alumina (pore volume of Al120-8h is approximately 1.8 times of that of commercially activated alumina), and A1120-8h displays a much better performance than that of commercially activated alumina.

It is hard to avoid undesirable polymerization of D4 siloxane on the surface of the adsorbent.[19] D4 siloxane can interact with hydroxyl groups on the adsorbent surface by hydrogen bonding, and surface hydroxyl groups can function as active catalytic centers for ring-opening polymerization of siloxane.[1,44,46] However, lower or higher molecular weight siloxanes are not detected after one run, which is beneficial for the longevity and regeneration of the adsorbents. Acidic and basic sites on Al120-8h are also related to the better adsorption performance induced by polymerization,[47] which can also explain the $15 \%$ capacity loss in the third recycle of the adsorbent. The $\mathrm{CO}_{2}-\mathrm{TPD}$ profiles and FTIR spectra of pyridine adsorption were recorded to study the differences in the types, strengths, and the amount of basic and acidic sites of the selected Al120-8h and commercially activated alumina. These profiles are shown in Figures $5 \mathrm{a}$ and $\mathrm{b}$, respectively. The $\mathrm{CO}_{2}-\mathrm{TPD}$ profile of MA Al120-8h in Figure 5a shows three peaks, indicating the existence of basic sites on the surface 
of the sample studied. Desorption peaks are centered at 100 and $299{ }^{\circ} \mathrm{C}$, while the main desorption peak of commercially activated alumina is located at $100{ }^{\circ} \mathrm{C}$. Since acidic $\mathrm{CO}_{2}$ desorbs at a higher temperature from stronger basic sites, and $\mathrm{CO}_{2}$ desorbs at a higher temperature $\left(299{ }^{\circ} \mathrm{C}\right)$ from Al120$8 \mathrm{~h}, \mathrm{Al120}-8 \mathrm{~h}$ has stronger basic sites.[48,49] The amount of desorbed $\mathrm{CO}_{2}$ from $\mathrm{Al120}-8 \mathrm{~h}$ is larger than that from commercially activated alumina when an equal amount of sample is tested, indicating Al120-8h has a larger quantity of basic sites. Figure 5b shows FTIR spectra of pyridine adsorbed alumina samples at room temperature. Pyridine adsorbed on surface hydroxyl groups $\left(1522 \mathrm{~cm}^{-1}\right)$ is labeled as $-\mathrm{OH}$ (The reason is that surface hydroxyl groups act as Brønsted acid sites under ex-situ test conditions.[50,51] The vibrations of adsorbed pyridine do not distinguish the acidity because the band at $1522 \mathrm{~cm}^{-1}$ does not change in wavenumber upon varying the acidity of the solid [52]). Pyridine adsorbed on Lewis acid sites $\left(1400 \mathrm{~cm}^{-1}\right.$ and $\left.1630 \mathrm{~cm}^{-1}\right)$ are labeled as L[52].Both materials contain Lewis acid sites. The IR absorbance intensity from Lewis acid sites of Al120-8h is higher than that of commercially activated alumina, indicating a larger amount of acid sites for Al120-8h. MA exhibits a higher amount of Brønsted acid sites accompanied by small amounts of Lewis acid sites.-Since aluminum ions are the center of acidity, the acidity profile observed may be due to the aluminum ion distribution of the sample. The coordination environment of aluminum ions in assynthesized Al120-8h was examined by ${ }^{27}$ Al MAS NMR spectrometry. The spectrum of A1120-8h displays three resonance signals at $\delta=5,35$, and 61 (Figure 5c). These lines are indicative of six-, five-, and four-coordinate metal centers, respectively.[40] The presence of five-coordinate aluminum ion centers may have significant catalytic activity as Lewis acid centers.[40]

The advantage of a wormhole-like pore structure is its highly interconnected pore system,[20,21] whose high porosities and large surface areas are fully utilized. A wormhole-like pore structure can have better diffusion of D4 siloxane given mass-transfer limitations.[53] This structure 
also enhances the accessibility of the D4 molecules to the active centers (acid-base sites on the adsorbent surface),[19,24,53] which may give rise to the excellent adsorption performance. This wormhole-like pore structure could be one of the reasons why a high adsorption capacity of D4 siloxane is obtained with MA A1120-8h.

\section{Conclusions}

In summary, a sol-gel based inverse micelle method with a non-polar solvent (1-butanol) and short reaction times (4-8 h) was successfully modified for the synthesis of MA. The reaction times, surfactant chain lengths, and calcination temperatures and hold times had independent effects on the textural properties (pore sizes, surface areas, and pore volumes) of MA. The reaction times and surfactants were selected to continuously adjust the pore sizes and pore volumes of MA, while the calcination temperatures and hold times adjusted the surface areas and pore volumes without affecting the average pore size. Characterization studies of a representative sample, Al120-8h, by low angle XRD, $\mathrm{N}_{2}$ sorption, TEM, $\mathrm{CO}_{2}$-TPD, and pyridine adsorption FTIR, showed that the material formed a uniform wormhole-like pore structure with a relatively high amount of acidic and basic sites on the surface. Furthermore, Al120-8h presented a high capacity $(168 \mathrm{mg} / \mathrm{g})$ compared to commercially activated alumina for D4 siloxane adsorption, which was due to its large mesopore volume. Reusability studies indicate that the as-synthesized MA is a promising adsorbent for the removal of siloxanes from biogas under ambient conditions.

\section{Acknowledgements}


The authors would like to acknowledge the support by Center of Energy Innovation and the UCONN Fraunhofer Center. The authors thank the UCONN-FEI Center for Advanced Microscopy and Materials Analysis. The authors thank Dr.You-Jun Fu for GC/MSD training, Drs. Marcus V. Giotto, Nicholas Eddy for NMR testing, and Dr. Francis Galasso, Dr. Ben Liu, Curtis Guild, Chris Monteleo for helpful discussions. 


\section{References}

[1] A. Cabrera-Codony, M. a. Montes-Morán, M. Sánchez-Polo, M.J. Martín, R. Gonzalez-Olmos, Environ. Sci. Technol. 48 (2014) 7187-7195.

[2] Y. Mito-oka, S. Horike, Y. Nishitani, T. Masumori, M. Inukai, Y. Hijikata, S. Kitagawa, J. Mater. Chem. A 1 (2013) 7885.

[3] N. de Arespacochaga, C. Valderrama, J. Raich-Montiu, M. Crest, S. Mehta, J.L. Cortina, Renew. Sustain. Energy Rev. 52 (2015) 366-381.

[4] L. Sigot, G. Ducom, B. Benadda, C. Labouré, Environ. Technol. 37 (2016) 86-95.

[5] A. Cabrera-Codony, R. Gonzalez-Olmos, M.J. Martín, J. Hazard. Mater. 285 (2015) 501-8.

[6] S. Du, Y. Sun, D.P. Gamliel, J.A. Valla, G.M. Bollas, Bioresour. Technol. 169 (2014) 188-197.

[7] S. Du, J. a. Valla, G.M. Bollas, Green Chem. 15 (2013) 3214.

[8] M. Schweigkofler, R. Niessner, J. Hazard. Mater. 83 (2001) 183-196.

[9] R. Dewil, L. Appels, J. Baeyens, Energy Convers. Manag. 47 (2006) 1711-1722.

[10] I. Noshadi, B. Kanjilal, T. Jafari, E. Moharreri, N. Khakpash, T. Jiang, S.L. Suib, RSC Adv. 6 (2016) 77310-77320.

[11] G. Soreanu, M. Béland, P. Falletta, K. Edmonson, L. Svoboda, M. Al-Jamal, P. Seto, Can. Biosyst. Eng. 53 (2011) 8.1-8.18.

[12] M. Yu, H. Gong, Z. Chen, M. Zhang, J. Environ. Chem. Eng. 1 (2013) 1182-1187.

[13] E. Finocchio, T. Montanari, G. Garuti, C. Pistarino, F. Federici, M. Cugino, G. Busca, Energy \& Fuels 23 (2009) 4156-4159.

[14] V.L. Higgins, Siloxane Removal Process, US7,306,652 B2, 2007.

[15] S. Nam, W. Namkoong, J.-H. Kang, J.-K. Park, N. Lee, Waste Manag. 33 (2013) 2091-8.

[16] J.-K. Park, G.-M. Lee, C.-Y. Lee, K.-B. Hur, N.-H. Lee, Environ. Eng. Res. 17 (2012) 117-122.

[17] E. Finocchio, G. Garuti, M. Baldi, G. Busca, Chemosphere 72 (2008) 1659-1663. 
[18] Y. Lee, Seung-Ho; Cho, Wonihl; Song, Taek-Yong; Kim, Hoyeon; Lee, Woo-Jin; Lee, YoungChul; Baek, J.Ind.Eng.Chem. 7 (2001) 276-280.

[19] T. Jiang, W. Zhong, T. Jafari, S. Du, J. He, Y.-J. Fu, P. Singh, S.L. Suib, Chem. Eng. J. (2016).

[20] S. Cabrera, J.E. Haskoiiri, J. Alamo, A. Beltrán, D. Beltrân, S. Mendioroz, M. Dolores Marcos, PedroAmorôs, Adv. Mater. 11 (1999) 379-381.

[21] Y. Kim, C. Kim, I. Choi, S. Rengaraj, J. Yi, Environ. Sci. Technol. 38 (2004) 924-931.

[22] S.M. Morris, P.F. Fulvio, M. Jaroniec, J. Am. Chem. Soc. 130 (2008) 15210-15216.

[23] P.F. Fulvio, R.I. Brosey, M. Jaroniec, ACS Appl. Mater. Interfaces 2 (2010) 588-593.

[24] W. Cai, J. Yu, C. Anand, A. Vinu, M. Jaroniec, Chem. Mater. 23 (2011) 1147-1157.

[25] Y. Wan, Y. Shi, D. Zhao, Chem. Commun. (Camb). (2007) 897-926.

[26] F. Vaudry, S. Khodabandeh, M.E. Davis, Chem. Mater. 8 (1996) 1451-1464.

[27] P. Kim, Y. Kim, H. Kim, I.K. Song, J. Yi, J. Mol. Catal. A Chem. 219 (2004) 87-95.

[28] K. Niesz, P. Yang, G. a Somorjai, Chem. Commun. (Camb). (2005) 1986-1987.

[29] N.D. Wasalathanthri, A.S. Poyraz, S. Biswas, Y. Meng, C.H. Kuo, D.A. Kriz, S.L. Suib, J. Phys. Chem. C 119 (2015) 1473-1482.

[30] A.S. Poyraz, W. Song, D. Kriz, C.-H. Kuo, M.S. Seraji, S.L. Suib, ACS Appl. Mater. Interfaces 6 (2014) 10986-10991.

[31] W. Song, A.S. Poyraz, Y. Meng, Z. Ren, S.Y. Chen, S.L. Suib, Chem. Mater. 26 (2014) 4629_ 4639.

[32] Z. Luo, A.S. Poyraz, C. Kuo, R. Miao, Y. Meng, S. Chen, T. Jiang, C. Wenos, S.L. Suib, Chem. Mater. 27 (2015) 6-17.

[33] R. Miao, Z. Luo, W. Zhong, S.-Y. Chen, T. Jiang, B. Dutta, Y. Nasr, Y. Zhang, S.L. Suib, Appl. Catal. B Environ. 189 (2016) 26-38. 
[34] T. Jiang, S. Du, T. Jafari, W. Zhong, Y. Sun, W. Song, Z. Luo, W. a. Hines, S.L. Suib, Appl. Catal. A Gen. 502 (2015) 105-113.

[35] T. Jiang, A.S. Poyraz, A. Iyer, Y. Zhang, Z. Luo, W. Zhong, R. Miao, A.M. El-Sawy, C.J. Guild, Y. Sun, D. a. Kriz, S.L. Suib, J. Phys. Chem. C 119 (2015) 10454-10468.

[36] A.S. Poyraz, C.-H. Kuo, S. Biswas, C.K. King'ondu, S.L. Suib, Nat. Commun. 4 (2013) 2952.

[37] D. Zhao, J. Feng, Q. Huo, N. Melosh, G. Fredrickson, B. Chmelka, G. Stucky, Science 279 (1998) $548-52$.

[38] A. Čejka , Jiří ; Žilková, Naděžda ; Rathouský, Jiří and Zukal, Phys. Chem. Chem. Phys. 3 (2001) $5076-5081$.

[39] F. Rashidi, A.N. Kharat, A.M. Rashidi, E. Lima, V. Lara, J.S. Valente, Eur. J. Inorg. Chem. (2010) $1544-1551$.

[40] S.A. Bagshaw, T.J. Pinnavaia, Angew. Chemie Int. Ed. English 35 (1996) 1102-1105.

[41] T. Jafari, I. Noshadi, N. Khakpash, S.L. Suib, J. Mater. Chem. A 3 (2015) 5023-5030.

[42] J.M.Bellama, J.Schraml, in:, F.C. Nachod (Ed.), Determ. Org. Struct. By Phys. Methods, Elsevier, 2012, pp. 203-268.

[43] T. Montanari, E. Finocchio, I. Bozzano, G. Garuti, A. Giordano, C. Pistarino, G. Busca, Chem. Eng. J. 165 (2010) 859-863.

[44] L. Sigot, G. Ducom, P. Germain, Microporous Mesoporous Mater. 213 (2015) 118-124.

[45] W. Cai, J. Yu, M. Jaroniec, J. Mater. Chem. 21 (2011) 9066.

[46] P. Soreanu, Gabriela; Béland, Michel; Fallettal, Patricia; Edmonson, Kara; Svoboda, Lewina; AlJamal, Mohamad; Seto, Can. Biosyst. Eng. 53 (2011) 8.1-8.18.

[47] M. Mitariten, Michael J.; Brown, Roy; Ballantyne, Wayne; Rossin, Joseph; Knapke, Regenerative Adsorption Process for Removal of Silicon-Containing Contaminants from Process Gas Using a Neutral Adsorbent Media, US 20140366726A1, 2014. 
[48] H.-Y.C. and W.-F.L. Yu-Wen Chen, React.Kinet.Catal.Lett. 65 (1998) 83-86.

[49] T. Seki, M. Onaka, J. Mol. Catal. A Chem. 263 (2007) 115-120.

[50] R.. Pearson, J. Catal. 46 (1977) 279-288.

[51] Y.F. Shen, S.L. Suib, M. Deeba, G.S. Koermer, J. Catal. 146 (1994) 483-490.

[52] K.A. Layman, M.M. Ivey, J.C. Hemminger, J. Phys. Chem. B 107 (2003) 8538-8546.

[53] G. Wang, L. Zhang, H. Dai, J. Deng, C. Liu, H. He, C.T. Au, Inorg. Chem. 47 (2008) 4015-22. 

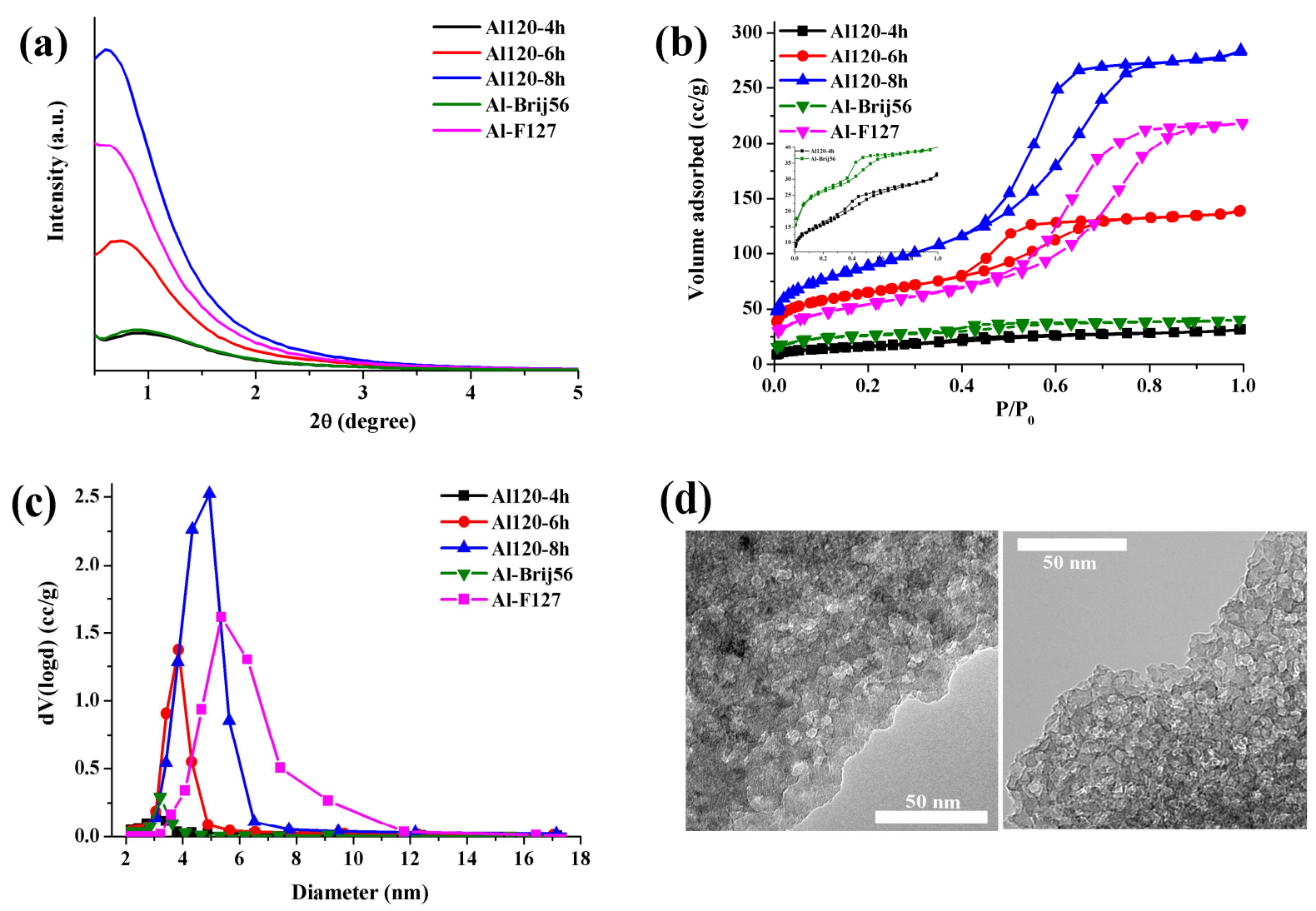

Figure 1. (a) Low angle XRD patterns (b) $\mathrm{N}_{2}$ sorption isotherm curves (inset: isotherm curves of Al1204h, Al-Brij56) and (c) pore size distributions of MA samples Al120-4h, Al120-6h, Al120-8h, Al-Brij56, and Al-F127. (d) TEM images of representative samples Al120-6h and Al120-8h. 

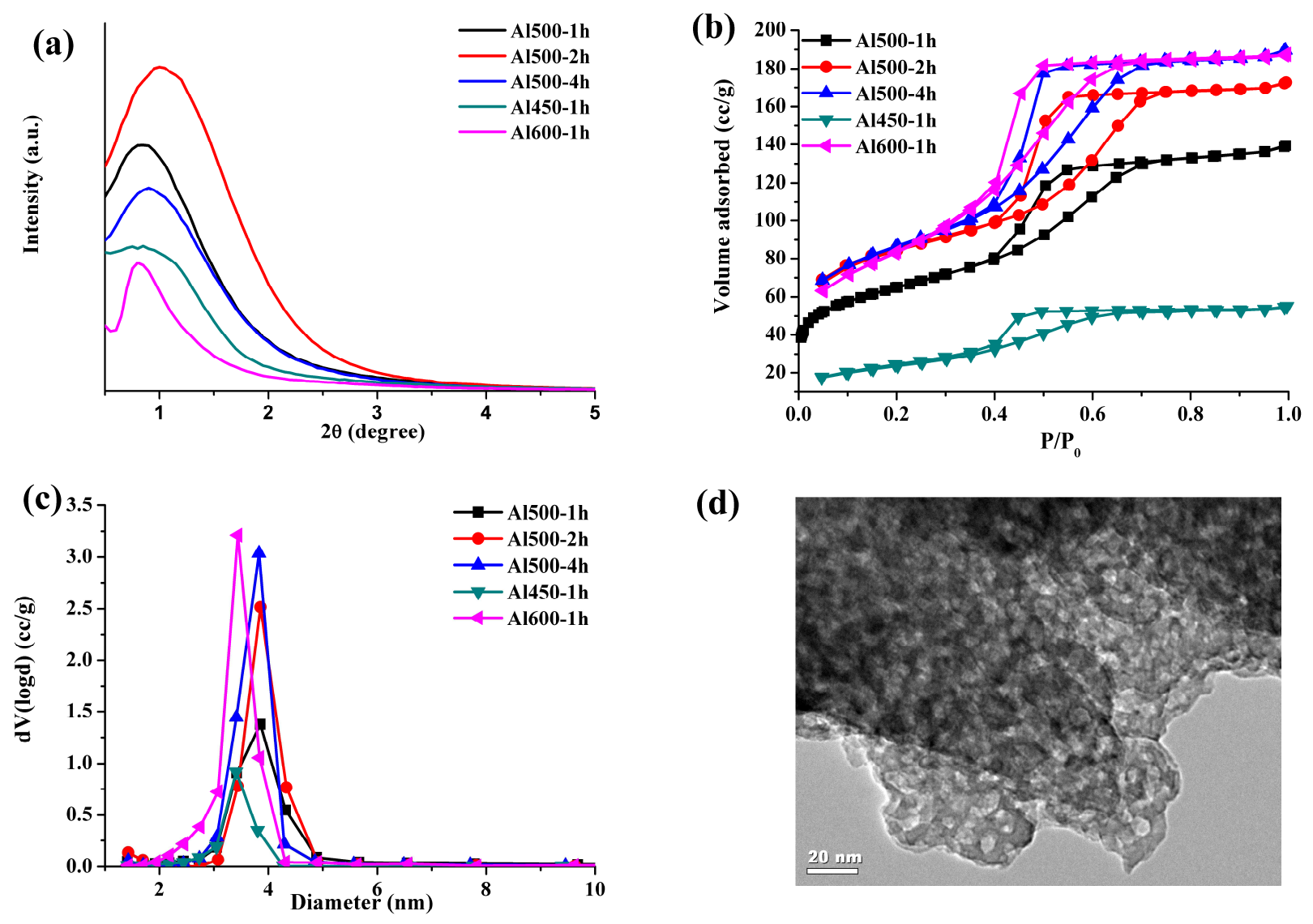

(d)

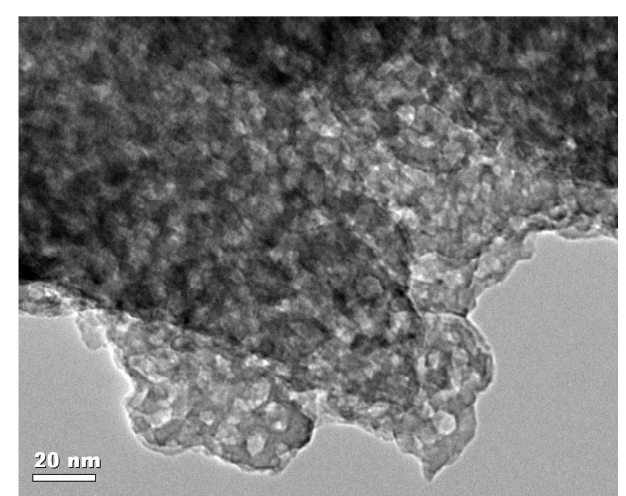

Figure 2. (a) Low angle X-ray diffraction patterns, (b) $\mathrm{N}_{2}$ sorption isotherm curves and (c) pore size distributions of MA samples Al500-1h, Al500-2h, Al500-4h, Al450-1h, and Al600-1h. (d) Transmission electron microscopy (TEM) image of A1500-1h. 
Table 1. Textural Properties of MAs of Different Reaction Times, Surfactants and Calcination Temperature and Hold Time.

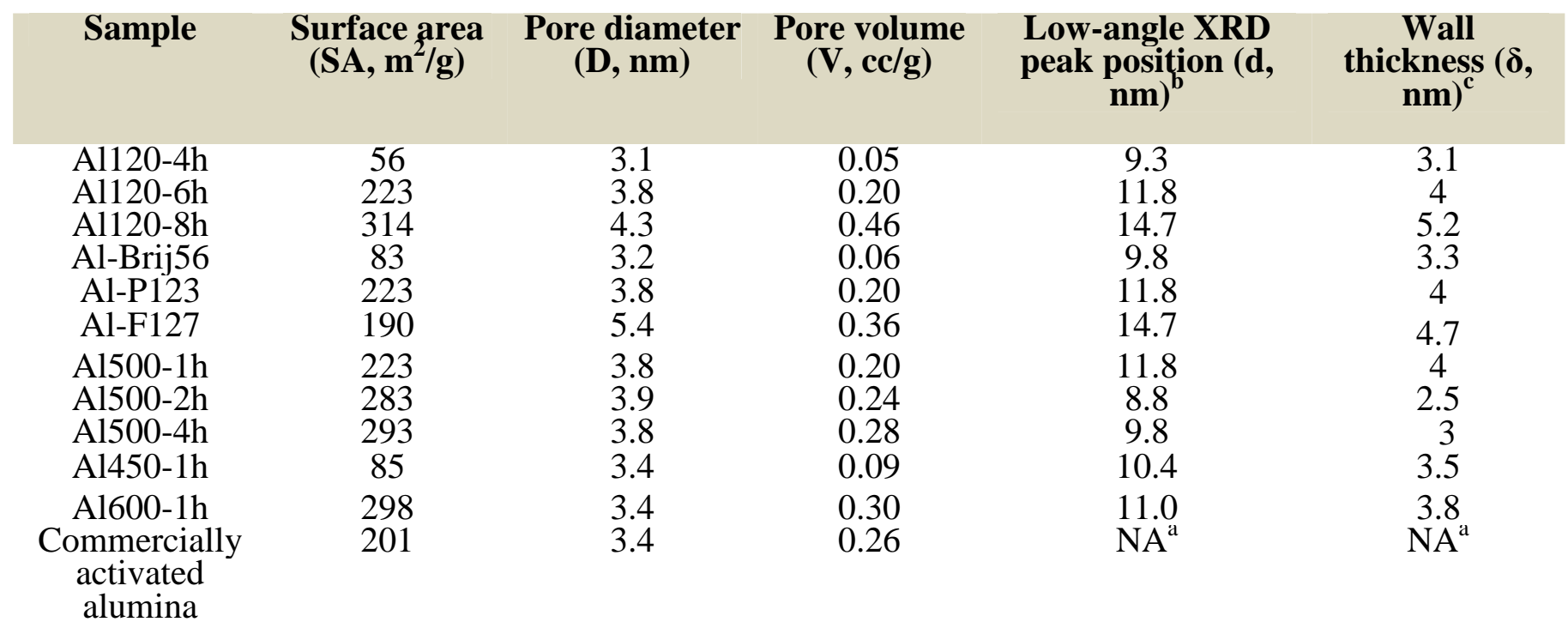

${ }^{\mathrm{a}}$ Not available. ${ }^{\mathrm{b}}$ Calculation is according to Bragg's law. ${ }^{\mathrm{c}}$ Wall thickness $\delta=(\mathrm{d}-\mathrm{D}) / 2$. 


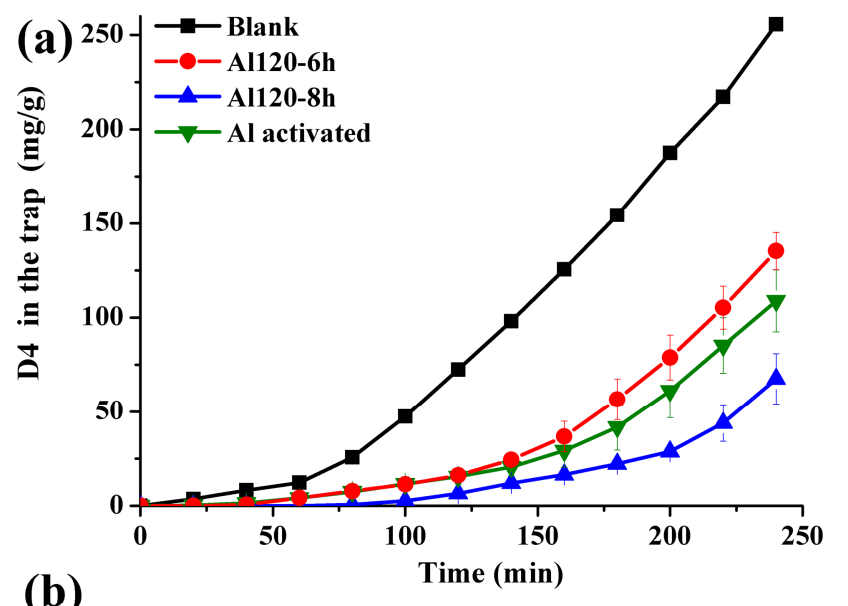

(b)

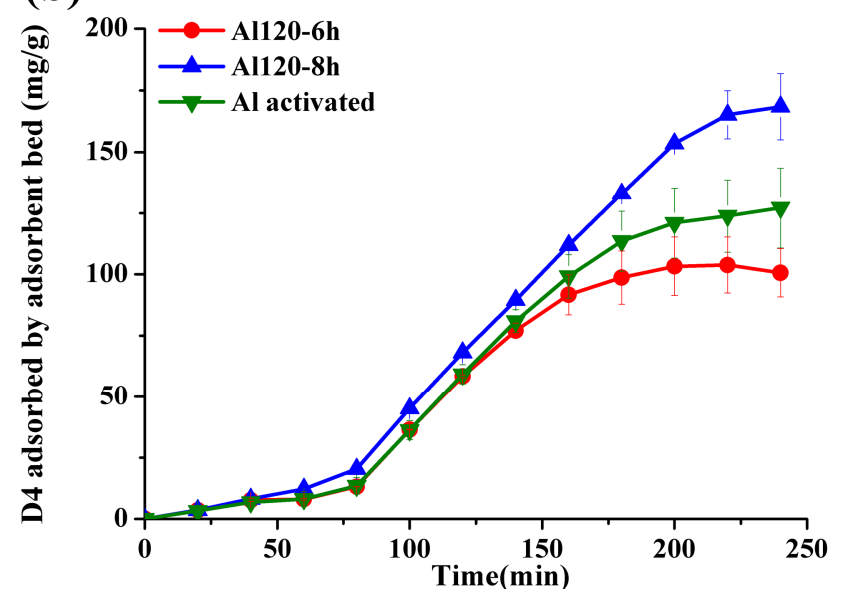

Figure 3. (a) Breakthrough curves obtained by plotting accumulated D4 siloxane in the hexane trap versus time. (b) Saturation curves obtained by plotting adsorbed D4 siloxane on adsorbents Al120-6h, Al120-8h, and commercially activated alumina. 

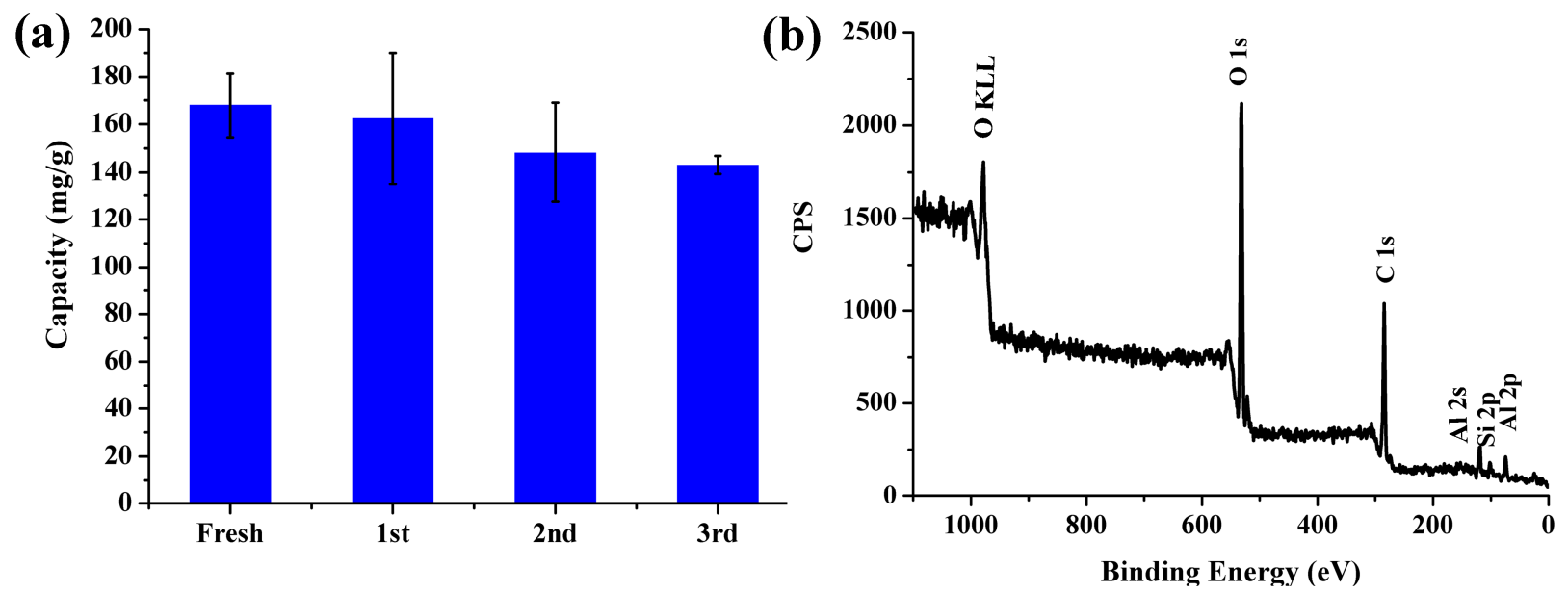

(c)
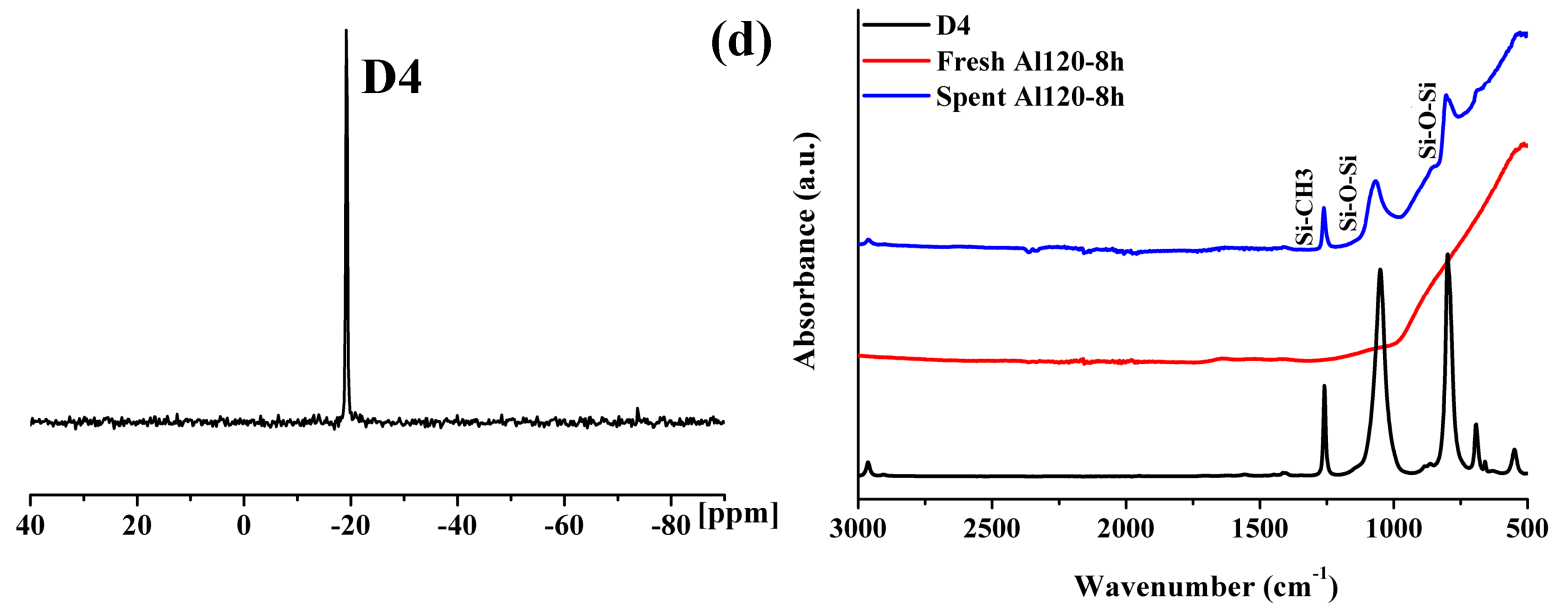

Figure 4. (a) Wash capacities of regenerated adsorbent Al120-8h. (b) XPS of a full-range spectrum, and (c) ${ }^{29}$ Si MAS NMR spectrum for spent adsorbent Al120-8h after one run. (d) ATR spectra of D4, fresh and spent Al120-8h after one run. 


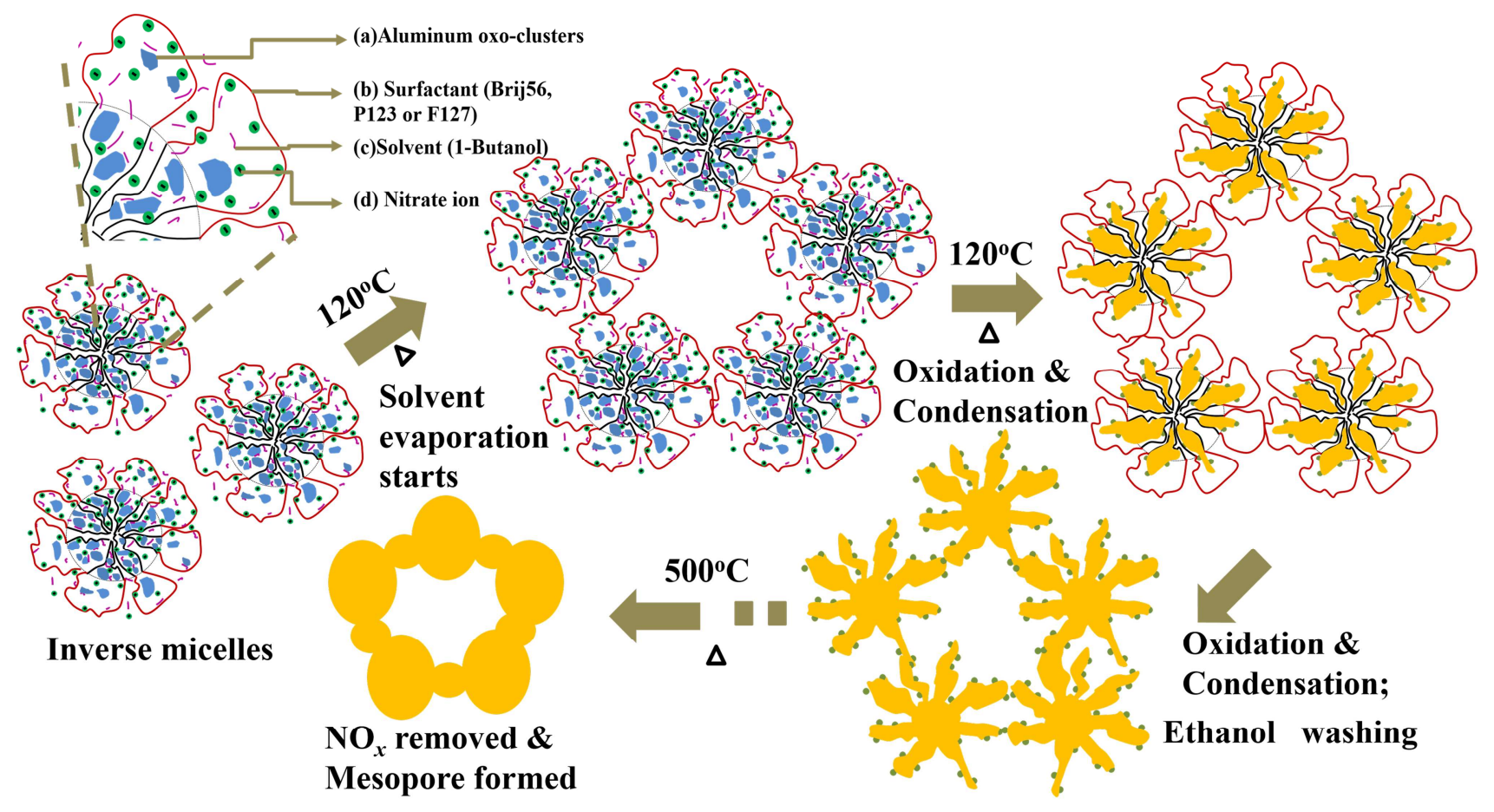

Scheme 1. Proposed formation mechanism of MA. 

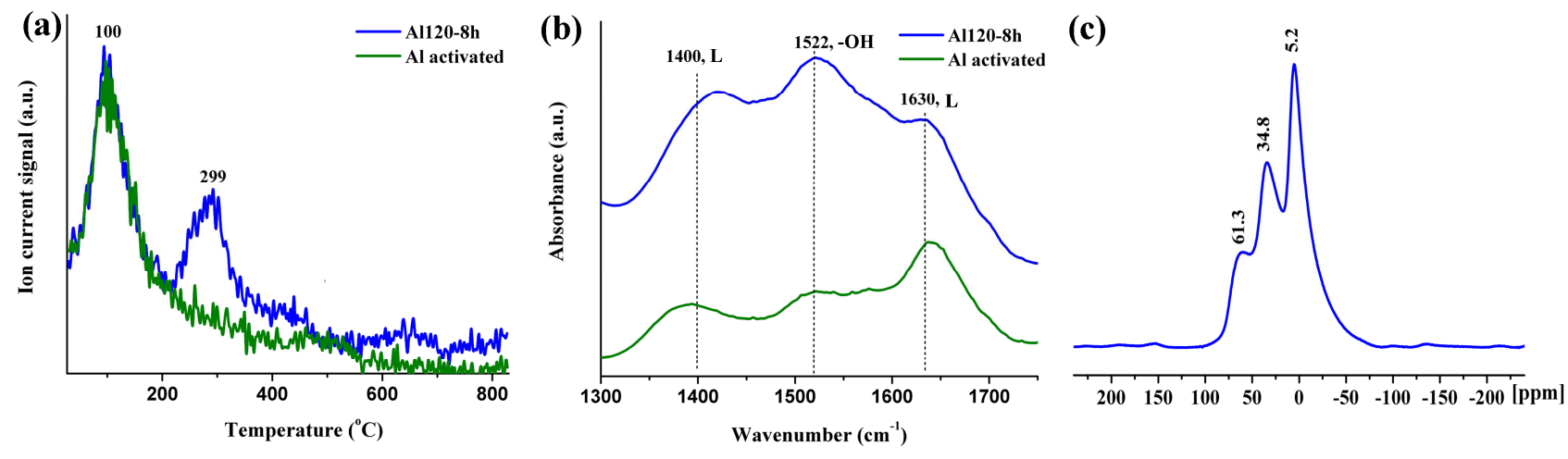

Figure 5. (a) $\mathrm{CO}_{2}$-TPD profiles and (b) pyridine adsorption FTIR spectra for $\mathrm{Al} 120-8 \mathrm{~h}$ and commercially activated alumina. (c) ${ }^{27}$ Al MAS NMR spectrum for as-synthesized Al120-8h. 


\section{Highlights:}

1. This is the first time the extension of the inverse micelle synthetic strategy to mesoporous alumina (MA) was used. Reaction times, surfactant chain lengths, and calcination temperatures and hold times were chosen as parameters to continuously adjust pore sizes, surface areas, and pore volumes of MA.

2. A reaction time of $8 \mathrm{~h}$ when P123 was chosen as the surfactant yielded a material with a high capacity (168 mg/g) for octamethylcyclotetrasiloxane (D4 siloxane), a 32\% capacity improvement compared to commercially available activated alumina. This is related to the large mesopore volume of this material.

3. Approximately $85 \%$ of D4 adsorption capacity can be maintained after three adsorption recycles, which indicates the as-synthesized MA is a promising adsorbent for the removal of D4 siloxane from biogas under ambient conditions. 